\title{
Argon Solvent Effects on Optical Properties of Silver Metal Clusters ${ }^{\dagger}$
}

\author{
W. Christen, ${ }^{\ddagger}$ P. Radcliffe, ${ }^{\S, \|}$ A. Przystawik, ${ }^{\S, \perp}$ Th. Diederich, ${ }^{\S}$ and J. Tiggesbäumker ${ }^{*, \S}$ \\ ${ }^{\ddagger}$ Institut für Chemie, Humboldt-Universität zu Berlin, Brook-Taylor-Str. 2, 12489 Berlin, Germany, and \\ §Institut für Physik, Universität Rostock, Universitätsplatz 3, 18051 Rostock, Germany
}

ABSTRACT: Argon gas at a high pressure ( 80 bar) has been expanded using a miniaturized pulsed valve at room temperature, producing a supersonic beam of cold, large argon droplets. Atoms of silver are subsequently embedded into the droplet using the pick-up technique. The resulting $\mathrm{Ag}_{n} \mathrm{Ar}_{\text {droplet }}$ distribution was analyzed using multiphoton laser ionization time-of-flight mass spectrometry. Besides bare metal clusters, snowballs of silver monomers and dimers encapsulated in up to 50 argon atoms have been observed. The influence of the solvent on the optical absorption of the solute was studied for embedded $\mathrm{Ag}_{8}$ using resonant two-photon ionization in the ultraviolet. A redshift and broadening of the $\mathrm{Ag}_{8} \mathrm{Ar}_{\text {droplet }}$ optical spectrum compared to that measured in pure [Federmann et al., Eur. Phys. J. D 1999, 9, 11] and Ar-doped helium droplets [Diederich et al., J. Chem. Phys. 2002, 116, 3263] was observed, which is attributed to the interaction with the larger Ar matrix environment.

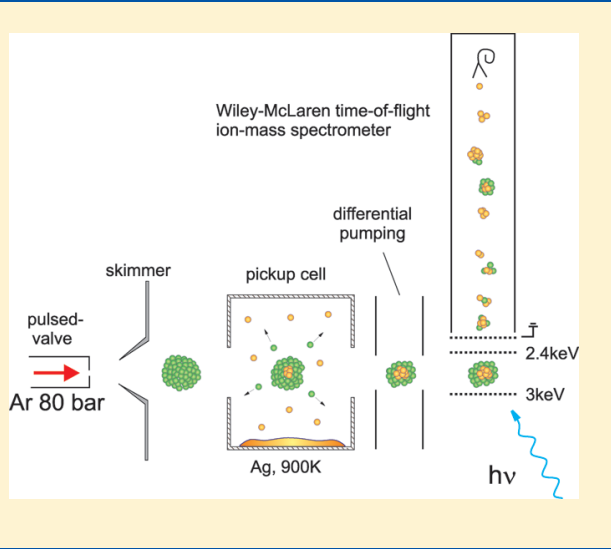

\section{INTRODUCTION}

One of the most fascinating aspects in small alkali and noble metal clusters is the evolution of electronic and structural properties, such as the transition from discrete, molecular toward collective, metallic characteristics as a function of particle size. ${ }^{1}$ Silver clusters are of special interest for a number of reasons. Due to the single $\mathrm{s}^{1}$ valence electron, individual $\mathrm{Ag}$ atoms in many cases can be treated in close analogy to alkali atoms. Therefore, it is possible to explain a variety of physical properties of silver clusters by comparatively simple theoretical approaches. Prominent examples include the particle abundance distribution and the size dependence of ionization potentials and electron affinities, see ref 2 for details.

It is well-known that electronic and optical properties of small aggregates strongly depend on the structure of the cluster. ${ }^{3}$ The particle charge state often modifies not only its physical and chemical properties but also its geometry. Hence, it is of significant interest to perform measurements both on the neutral and charged species. In general, the need for mass-selection due to the broad neutral size distribution usually delivered by cluster sources, in combination with the short lifetime of intermediate states in metallic systems, render such experiments difficult. However, in $\mathrm{Ag}_{n}$, a number of optical transitions with an excited state lifetime in the nanosecond range have been identified. These make laser-induced fluorescence (LIF) ${ }^{4}$ and resonant twophoton ionization (R2PI) measurements with nanosecond laser pulses ${ }^{5,6}$ feasible. In attempting to study not only mass-selected species but also clusters of a given geometry, preselection of the energetically lowest lying isomer in a molecular beam is essential. This goal can be approached for clusters at particularly low temperatures. An effective way of cooling hot metal clusters is embedding them into cold and inert rare gas matrices. Accordingly, metal clusters in rare gas matrices and, even better defined, in rare gas droplets, have developed into an intensely investigated model system. ${ }^{5,7-9}$ Employing large rare gas clusters, very low temperatures of the metal clusters can be achieved; for example, temperatures as low as $0.37 \mathrm{~K}$ can be provided in helium nanodroplets, ${ }^{10}$ but also, for other rare gases, temperatures of only a few Kelvin have been reported. ${ }^{11,13,14}$

To gain an independent control both on the mean droplet size as well as the metal cluster formation process, the pick-up technique can be applied, first demonstrated by the groups of Scoles ${ }^{15}$ and Toennies. ${ }^{16}$ This experimental approach provides a soft matrix for embedded species, minimizing inhomogeneous line broadening. Recently, clusters with up to 1500 atoms ${ }^{17}$ and even proteins ${ }^{18}$ have been successfully loaded into large helium nanodroplets using this method. Because pulsed laser systems are frequently used for excitation and ionization, there is a strong need for a high target density in the interaction region where the laser pulse crosses the molecular beam. Vilesov and co-workers have shown that a modified source based on the model 99 from General Valve can be used to generate a pulse of cold nanodroplets of helium doped with phthalocyanine. ${ }^{19}$ Simultaneously, the flux has been increased by a factor of up to 100 compared to continuously working helium droplet sources. ${ }^{20}$ In this contribution we focus on the pick-up of silver atoms to form clusters using a pulsed jet expansion of argon with a valve opening time of only $20 \mu \mathrm{s}$, thus, minimizing the gas load substantially. We report on the generation and mass spectrometric detection of cold medium-sized silver clusters embedded in argon rare gas droplets.

The effect of temperature and solvent interaction on the cluster is of considerable interest on its own. ${ }^{21}$ It is, thus,

Received: January 17, 2011

Revised: June 15, 2011 


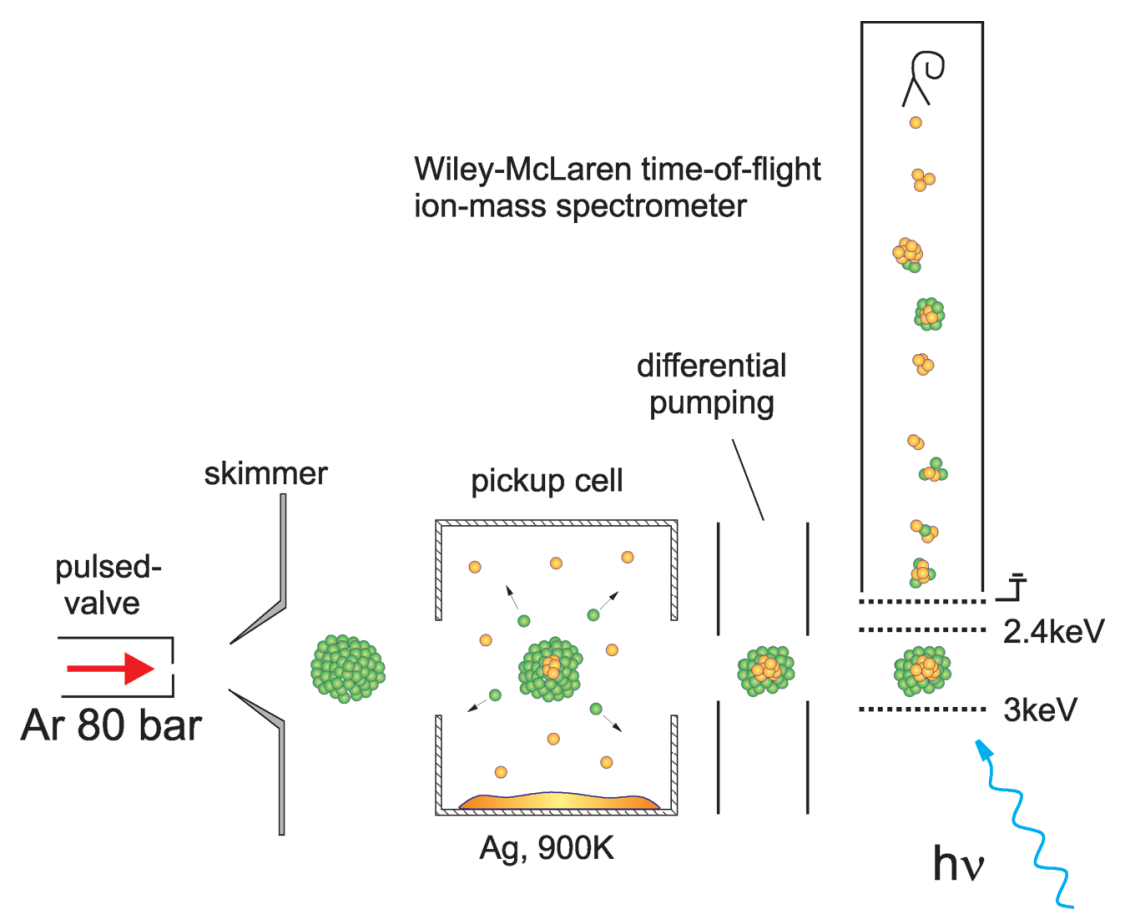

Figure 1. Schematic diagram of the molecular beam apparatus used to generate silver-doped argon droplets. The droplets are formed in a high-pressure supersonic jet expansion of room temperature Ar from a pulsed nozzle backed at 80 bar. Silver atoms are loaded into the droplets via a $5 \mathrm{~cm}$ long pick-up stage where they coalesce to form $\mathrm{Ag}_{n}$ clusters. Upon multiphoton ionization using a pulsed nanosecond ultraviolet laser the cluster ions are accelerated into a Wiley-McLaren time-of-flight mass spectrometer.

worthwhile to investigate the optical absorption of silver clusters embedded in different rare gas droplets. In this work we demonstrate an application of our cold source of argon droplets in a proof-of-principle experiment by measuring the optical response of embedded $\mathrm{Ag}_{8}$ in a resonant two-photon ionization scheme. We compare the results to previous studies on $\mathrm{Ag}_{8}$ in pure and argon-doped helium droplets as well as pure argon droplets.

\section{EXPERIMENTAL SECTION}

Argon droplets are produced in a high-pressure supersonic jet expansion of high purity Ar gas (99.9999\%) through a miniaturized pulsed valve (Even-Lavie setup) ${ }^{11}$ at room temperature and typical stagnation pressures of $P_{0}=80$ bar. This high-pressure expansion facilitates very low temperatures in the expanding jet, ${ }^{22,23}$ while the short opening time of $20 \mu$ s reduces the gas load considerably compared to other systems (e.g., General Valve, model 99). It permits operation of the cluster source with a single pumping stage of $\sim 900 \mathrm{ls}^{-1}$, with $10^{12}-10^{14}$ atoms per pulse depending on the backing pressure. ${ }^{24}$ Argon droplets of a mean size of $2 \times 10^{4}$ are produced. The mean cluster size has been estimated using well-known scaling laws where the average cluster size is supposed to only depend on expansion conditions, that is, source pressure, source temperature, and nozzle shape and dimensions. ${ }^{12}$ The expanding jet is collimated by a skimmer and traverses a $5 \mathrm{~cm}$ long region of increased silver vapor pressure $\left(10^{-5}-10^{-4} \mathrm{mbar}\right)$, resulting from a resistively heated oven operated at approximately $1100 \mathrm{~K}$ where the pick-up process takes place. Subsequently, a few centimeters downstream of the oven, the neutral clusters are ionized by nanosecond pulses from a Nd:YAG pumped dye laser system (Quanta Ray, LAS) operated at $20 \mathrm{~Hz}$ repetition rate. The resulting ions are accelerated perpendicular to the molecular beam axis; their size distribution is recorded using a linear time-of-flight mass spectrometer (TOFMS), as shown in Figure 1. The extraction unit consists of a twostage Wiley-McLaren type acceleration with a constant voltage of $3000 \mathrm{~V}$ applied to the back plate. Employing multiphoton ionization of the silver cluster-doped argon droplets, mass spectra could be recorded with this setup. Using a confined laser focus, the start volume in the TOF-MS is well-defined, permitting a mass resolution of about 150 .

\section{RESULTS AND DISCUSSION}

Silver-Argon Complex Formation. To characterize the neutral mass distribution, electron impact ionization (EII) or laser ionization can be applied. In EII the droplet is first charged and the embedded complex is subsequently ionized via charge transfer, releasing a high amount of excess energy (see, e.g., ref 25). With ultraviolet laser excitation, the photon energy is directly deposited into the dopant and the experimental mass spectra should give a more realistic image of the beam composition compared to EII. Thus, for a first characterization of the mass distribution of the neutral complexes formed in the pick-up process, multiphoton ionization was applied using laser pulses at a wavelength of $313.5 \mathrm{~nm}$. Depending on the target density in the pick-up region, the ion mass spectra show contributions from different species. For example, at high vapor pressures, clusters with up to 10 silver atoms are detected, indicating metal cluster growth. The size distribution (not shown) exhibits the typical odd-even alternation, see ref 2 for other examples. The bare metal clusters have been formed with sufficient internal energy to evaporate all argon atoms. The lack of $\mathrm{Ag}_{4}^{+}$and $\mathrm{Ag}_{6}^{+}$, as well as the pronounced peak intensities of $\mathrm{Ag}_{2}^{+}$and $\mathrm{Ag}_{7}^{+}$, resemble the laser ionization spectrum of Federmann et al., ${ }^{5}$ obtained using helium as the droplet environment. In addition, $\operatorname{Ag}_{n} \operatorname{Ar}_{m}(n=1-3)$ ion 


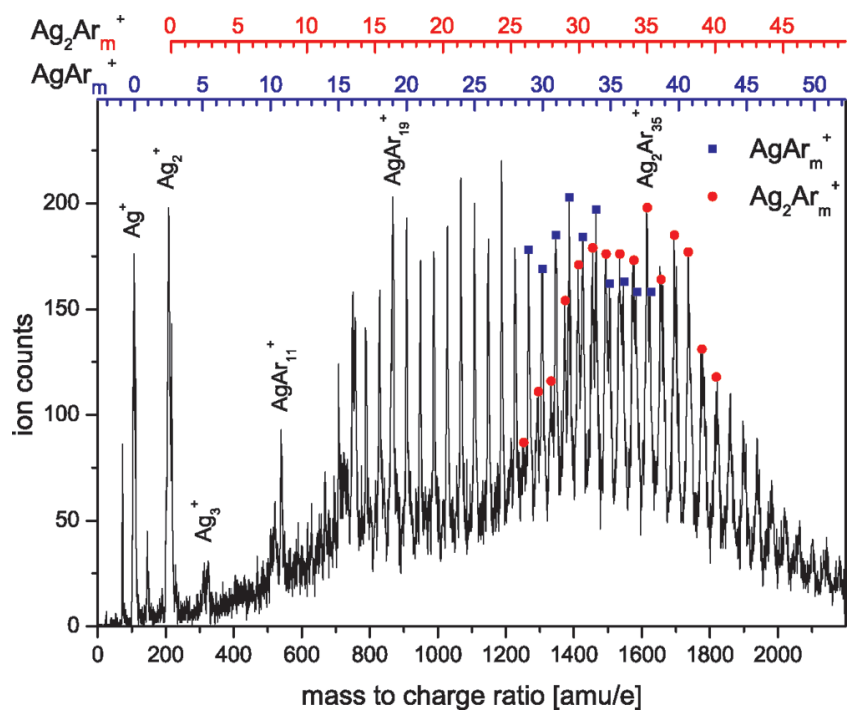

Figure 2. Mass spectrum of silver-argon cluster cations where the source conditions were tuned to generate mostly $\mathrm{AgAr}_{m}^{+}$. In addition, argon-coated silver dimers, $\mathrm{Ag}_{2} \mathrm{Ar}_{m}^{+}$(circles) are present, too. For convenience, the position of some $\mathrm{AgAr}_{m}^{+}$are indicated to visualize the shift in the yields from $\mathrm{AgAr}_{m}^{+}$(squares) to $\mathrm{Ag}_{2} \mathrm{Ar}_{m}^{+}$at around $1.600 \mathrm{amu}$.

snowball complexes are present. At lower metal vapor pressures, the $\mathrm{Ag}_{n}^{+}$distribution shifts to smaller sizes, as expected; accordingly, the intensity of the clusters containing only one or two silver atoms, $\mathrm{Ag}^{+} \mathrm{Ar}_{m}$ and $\mathrm{Ag}_{2}^{+} \mathrm{Ar}_{m}$, respectively, increases. $\mathrm{A}$ typical mass spectrum of $\mathrm{Ag}^{+} \mathrm{Ar}_{m}$ snowballs is shown in Figure 2. $\mathrm{The} \mathrm{AgAr}_{m}$ ion distribution extends up to about $m=50$. For $\mathrm{Ag}$ dimer and trimer snowballs, the size distribution narrows. In addition, the mass spectrum also hints at the smallest number $m_{c}$ of $\mathrm{Ar}$ atoms attached to $\mathrm{Ag}^{+}\left(m_{c}>10\right)$, which also appears to shift to larger values, for example, $<20$ for $\mathrm{Ag}_{2}^{+} \mathrm{Ar}_{m}$, reflecting the complex formation when using the pick-up technique. However, at present, no detailed studies have been performed to further quantify $m_{c}$ for the various ionic cores.

Due to the interaction with the laser light, the cluster size distribution is modified; excess energy from the ionization process is released into the cluster, heating the complex, which eventually may result in fragmentation. Hence, the observed abundance spectrum does not provide a one-to-one map of the original size distribution. In this experiment the neutral cluster formation originates from a two-step process: With respect to the stagnation conditions, a beam of argon droplets having a log-normal size distribution is generated, with translational temperatures as low as $1 \mathrm{~K}^{11} \mathrm{~A}$ collision between an $\mathrm{Ar}$ droplet and a silver atom may lead to the capture of the metal atom. Species picked up in this way are thermalized through collisions with the argon environment, which is acting as a heat sink. Excess energy, resulting from metal cluster formation, is dissipated into the rare gas droplet, leading to the evaporation of argon atoms. Approximately 100 atoms are evaporated per eV of released binding energy. Compared to similar experiments in helium droplets, ${ }^{10}$ these complexes will have temperatures above those of the initial rare gas droplets. As a rough estimate, the $\mathrm{Ar}_{2}$ dimer binding energy of $3 \mathrm{meV}^{26}$ could be taken as a reference for the temperature of the system. At higher oven temperatures successive pick-up events become possible, with the metal atoms coagulating to clusters. Their size distribution strongly depends on both the stagnation pressure and the vapor pressure in the

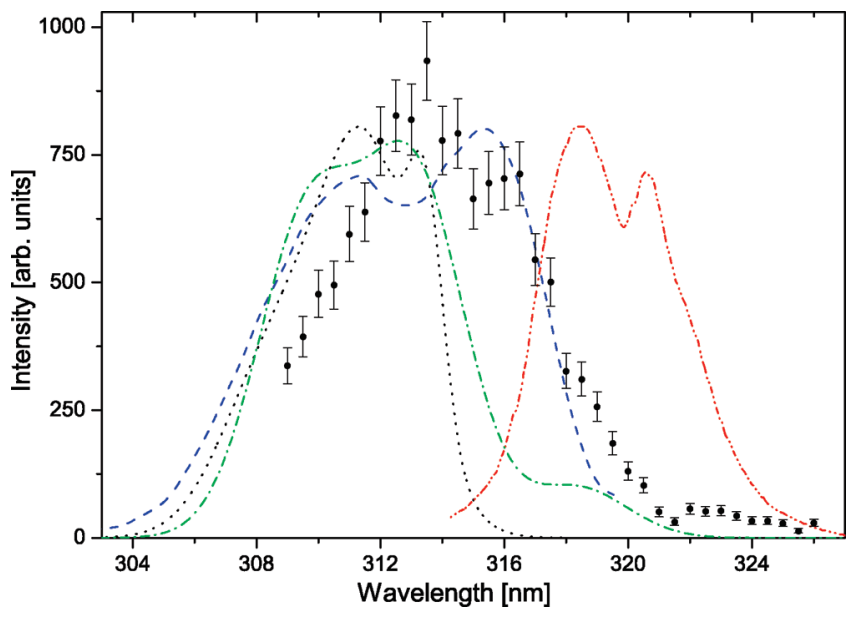

Figure 3. Optical spectra of $\mathrm{Ag}_{8}$ in argon droplets (black circles) obtained after single pulse resonant two-photon ionization, compared to previous work (black dotted line: $\mathrm{Ag}_{8}$ in $\mathrm{He}_{\text {droplets }}{ }^{21}$; green dasheddotted line $\mathrm{Ag}_{8}$ in $\mathrm{Ar}_{46} \mathrm{He}_{\text {droplets }}{ }^{21}$; blue dashed line: $\mathrm{Ag}_{8}$ in $\mathrm{Ar}$ matrix (excitation spectrum) ${ }^{4}$; red dashed-dotted-dotted line: $\mathrm{Ag}_{8}$ in $\mathrm{Ar}$ matrix (fluorescence spectrum $)^{4}$ ).

oven. Due to the two-step cluster formation process, the pick-up technique permits fine-tuning of both the complex size and the composition.

Optical Properties. The formation of metal clusters in the pick-up process allows to conduct additional experiments to study the optical properties of the $\mathrm{Ag}_{n} \mathrm{Ar}_{\text {droplet }}$ system. As a first case study, $\mathrm{Ag}_{3}$ was chosen, which is known to have an excited state suitable for selective nanosecond resonant two-photon ionization. ${ }^{27}$ Indeed, conducting such an experiment, $\mathrm{Ag}_{3}$ shows up in the mass spectrum with a high yield when laser photons of $340 \mathrm{~nm}$ are fed into the interaction volume to excite the beam (not shown here). Signals from the silver monomer and the dimer are also present. However, these are known to mostly result from trimer fragmentation. ${ }^{5}$ We note that some larger clusters also show evidence for long living states, that is, $\mathrm{Ag}_{4}{ }^{28}$ and $\mathrm{Ag}_{9}{ }^{29}$

To enable the comparison with former work, ${ }^{4,5,9,21}$ we have also investigated the silver octamer, which can be grown at higher vapor pressures. Figure 3 proves that $\mathrm{Ag}_{8}$ embedded in $\mathrm{Ar}$ droplets can be resonantly excited around $314 \mathrm{~nm}$. Due to the limited dye laser range, spectra were only recorded down to $309 \mathrm{~nm}$. Within a spectral range of more than $10 \mathrm{~nm}$ an enhanced yield of selectively ionized $\mathrm{Ag}_{8}$ can be detected. Taking into account a possible matrix shift, the feature can be identified by comparing to results from quantum chemical calculations. ${ }^{30,31}$ These have identified the corresponding multielectron resonances and a structure with $T_{\mathrm{d}}$ symmetry was suggested to be the ground state geometry, see, for example, ref 30 . We compare our measurements (circles) to experiments where the octamer is embedded in pure ${ }^{5}$ (black dotted line) and Ar-doped helium nanodroplets $^{21}$ (green dashed-dotted line). In the He droplet studies, the line width of the resonance is only about $56 \mathrm{meV} .^{5}$ This value was found to be substantially lower than the value obtained from the measurements on hot sputtered Ag cluster ions. ${ }^{32}$ In argon droplets we estimate a value of about $100 \mathrm{meV}$. In addition, a spectral redshift of the resonance compared to the helium result is obtained, which results from the interaction with the solvent. ${ }^{33}$ However, by comparing this study to ref 21 , there is evidence that the shift is influenced by the size of the Ar matrix, 
which is substantially larger in the pure argon droplet experiment. With respect to the excitation spectrum of ref 4 (blue dashed line), both the position as well as the line width agree well with the excitation spectra obtained for the argon matrix isolated octamer. Comparison with the matrix absorption spectrum of $\mathrm{Ag}_{8}$ in solid $\mathrm{Ar}^{34}$ reveals the likely existence of additional, short-lived multielectron states, which cannot be identified by LIF and nanosecond R2PI, leaving room for future work utilizing ultrashort excitation schemes.

\section{CONCLUSION}

A pulsed supersonic beam of cold neutral Ag clusters has been produced using pick-up of silver atoms by an argon droplet beam generated in a high pressure jet expansion using the Even-Lavie valve. The advantage of this particular technique is that the target composition, that is, the size of the droplets and of the embedded metal clusters can be adjusted independently by varying the rare gas backing pressure and the metal partial pressure in the pick-up cell. The method can be extended to other rare gases and even molecular species and allows for experiments on nanosolvents directly in the gas phase. ${ }^{35,36}$ As other possible applications, metal cluster soft-landing and cluster-surface scattering experiments are conceivable. Resonant two-photon ionization on the embedded complexes has proven that optical properties of embedded metal clusters can be probed. The optical spectra of $\mathrm{Ag}_{8}$ show a good agreement with other work on the same system but different matrix composition. In future experiments, the influence of a finite environment could thus be studied aiming to access the local influence of the solvent on the electronic properties of the solute.

\section{AUTHOR INFORMATION}

\section{Corresponding Author}

*E-mail: josef.tiggesbaeumker@uni-rostock.de.

\section{Present Addresses}

"European XFEL GmbH, Albert-Einstein-Ring 19, 22761 Hamburg, Germany.

${ }^{\perp}$ HASYLAB at DESY, Notkestr. 85, 22607 Hamburg, Germany.

\section{Notes}

'Originally submitted for the "J. Peter Toennies Festschrift", published as the June 30, 2011, issue of J. Phys. Chem. A (Vol. 115, No. 25).

\section{ACKNOWLEDGMENT}

We thank the Deutsche Forschungsgemeinschaft for financial support through Sonderforschungsbereich 652 . The authors gratefully acknowledge stimulating disussions with Karl-Heinz MeiwesBroer. W. Christen would like to express his gratitude to Professor Uzi Even for the generous donation of a room-temperature version of the pulsed valve. The valve driver was provided through a grant from the Deutsche Forschungsgemeinschaft ( $\mathrm{CH} 262 / 2)$.

\section{REFERENCES}

(1) Metal Clusters; Ekardt, W., Ed.; Wiley series in theoretical chemistry; John Wiley \& Sons Ltd: Chichester, 1999.

(2) de Heer, W. Rev. Mod. Phys. 1993, 65, 611.

(3) Häkkinen, H.; Moseler, M.; Kostko, O.; Morgner, N.; Hoffmann, M. A.; v. Issendorff, B. Phys. Rev. Lett. 2004, 93, 093401.
(4) Félix, C.; Sieber, C.; Harbich, W.; Buttet, J.; Rabin, I.; Schulze, W.; Ertl, G. Phys. Rev. Lett. 2001, 86, 2992.

(5) Federmann, F.; Hoffmann, K.; Quaas, N.; Toennies, J.-P. Eur. Phys. J. D 1999, 9, 11.

(6) Przystawik, A.; Radcliffe, P.; Göde, S.; Meiwes-Broer, K. H.; Tiggesbäumker, J. J. Phys. B 2006, 39, 1183.

(7) Bartelt, A.; Close, J.; Federmann, F.; Quaas, N.; Toennies, J.-P. Phys. Rev. Lett. 1996, 77, 3525.

(8) Haslett, T. L.; Bosnick, K. A.; Moskovits, M. J. Chem. Phys. 1998, 108, 3453.

(9) Radcliffe, P.; Przystawik, A.; Diederich, T.; Döppner, T.; Tiggesbäumker, J.; Meiwes-Broer, K.-H. Phys. Rev. Lett. 2004, 92, 173403.

(10) Hartmann, M.; Miller, R.; Toennies, J.-P.; Vilesov, A. Phys. Rev. Lett. 1995, 75, 1566.

(11) Even, U.; Jortner, J.; Noy, D.; Lavie, N.; Cossart-Magos, C. J. Chem. Phys. 2001, 112, 8068.

(12) Kim, K. Y.; Kumarappan, V.; Milchberg, H. M. Appl. Phys. Lett. 2003, 83, 3210.

(13) Hahns, M. Y.; Whetten, R. L. Phys. Rev. Lett. 1988, 61, 1190.

(14) Jellinek, J.; Beck, T. L.; Berry, R. S. J. Chem. Phys. 1986, 84, 2783.

(15) Goyal, S.; Schutt, D.; Scoles, G. Phys. Rev. Lett. 1992, 69, 933.

(16) Scheidemann, A.; Toennies, J.-P.; Northby, J. Phys. Rev. Lett. 1990, 64, 1899.

(17) Tiggesbäumker, J.; Stienkemeier, F. Phys. Chem. Chem. Phys. 2007, 9, 4748.

(18) Bierau, F.; Kupser, P.; Meijer, G.; von Helden, G. Phys. Rev. Lett. 2010, 105, 133402.

(19) Slipchenko, M.; Kuma, S.; Momose, T.; Vilesov, A. Rev. Sci. Instrum. 2002, 73, 3600.

(20) Pentlehner, D.; Riechers, R.; Dick, B.; Slenczka, A.; Even, U.; Lavie, N.; Brown, R.; Luria, K. Rev. Sci. Instrum. 2009, 80, 043302.

(21) Diederich, T.; Tiggesbäumker, J.; Meiwes-Broer, K.-H. J. Chem. Phys. 2002, 116, 3263.

(22) Hillenkamp, M.; Keinan, S.; Even, U. J. Chem. Phys. 2003, 118,8699 .

(23) Christen, W.; Rademann, K.; Even, U. J. Chem. Phys. 2006, $125,174307$.

(24) Christen, W.; Rademann, K. Rev. Sci. Instrum. 2006, 77, 015109.

(25) Callicoatt, B.; Förde, K.; Ruchti, T.; Jung, L.; Janda, K.; Halberstadt, N. J. Chem. Phys. 1998, 108, 9371.

(26) Ruzsinszky, A.; Perdew, J. P.; Csonka, G. I. J. Phys. Chem. A 2005, 109, 11015.

(27) Przystawik, A.; Radcliffe, P.; Diederich, T.; Döppner, T.; Tiggesbäumker, J.; Meiwes-Broer, K.-H. J. Chem. Phys. 2007, 126, 184306.

(28) Félix, C.; Sieber, C.; Harbich, W.; Buttet, J.; Rabin, I.; Schulze, W.; Ertl, G. Chem. Phys. Lett. 1999, 313, 105.

(29) Sieber, C.; Buttet, J.; Harbich, W.; Félix, C.; Mitric, R.; BonačićKoutecký, V. Phys. Rev. A 2004, 70, 041201.

(30) Bonačić-Koutecký, V.; Veyret, V.; Mitric, R. J. Chem. Phys. 2001, 115, 10450 .

(31) Tiago, M. L.; Idrobo, J. C.; Ögüt, S.; Jellinek, J.; Chelikowsky, J. R. Phys. Rev. B 2009, 79, 155419.

(32) Tiggesbäumker, J.; Köller, L.; Meiwes-Broer, K.-H.; Liebsch, A. Phys. Rev. A 1993, 48, R1749.

(33) Fedrigo, S.; Harbich, W.; Buttet, J. Int J. Mod. Phys. B 1992, 23-24, 3767.

(34) Harbich, W.; Fedrigo, S.; Buttet, J. Z. Phys. D 1993, 26, 138.

(35) Christen, W.; Geggier, S.; Grigorenko, S.; Rademann, K. Rev. Sci. Instrum. 2004, 75, 5048.

(36) Christen, W.; Krause, T.; Rademann, K. Int. J. Mass Spectrom. 2008, 277, 305. 